\title{
Interleukin 6: Biological significance and role in inflammatory bowel diseases
}

\author{
Agnieszka Pawłowska-Kamieniak ${ }^{\mathrm{A}, \mathrm{C}-\mathrm{E}}$, Paulina Krawiec ${ }^{\mathrm{A}, \mathrm{E}}$, Elżbieta Pac-Kożuchowska ${ }^{\mathrm{E}, \mathrm{F}}$ \\ Department of Pediatric and Gastroenterology, Medical University of Lublin, Poland \\ A - research concept and design; B - collection and/or assembly of data; $C$ - data analysis and interpretation; \\ $\mathrm{D}$ - writing the article; $\mathrm{E}$ - critical revision of the article; $\mathrm{F}$ - final approval of the article
}

Address for correspondence

Agnieszka Pawłowska-Kamieniak

E-mail: agnieszkapawlowskakamieniak@umlub.pl

Funding sources

None declared

Conflict of interest

None declared

Received on July 4, 2020

Reviewed on September 23, 2020

Accepted on November 11, 2020

Published online on April 28, 2021

\begin{abstract}
Cytokines affect a number of processes in the living body. Interleukin 6 (IL-6) is a cytokine involved in inflammation, infection response and also regulation of metabolism. It stimulates target cells through a membranebound IL-6 receptor. Inflammatory bowel diseases (IBD) are autoimmune diseases whose incidence and prevalence are increasing worldwide. It is a group of chronic gastrointestinal disorders characterized by multifactorial, still unknown pathogenesis, varied symptomatology, course with periods of exacerbation and remission, and polymorphic infiltration in histopathological examination. As it is known, pro-inflammatory cytokines, including IL-6, in IBD initiate, intensify and support the development of the inflammatory process in the intestine. Our knowledge of IL-6 biology has important consequences for therapeutic strategies. Elevation of IL-6 concentration can be considered as an early and sensitive, although non-specific marker for various inflammatory conditions and may be used in the diagnosis and monitoring of patients with IBD.
\end{abstract}

Key words: inflammatory bowel disease, pro-inflammatory cytokines, interleukin 6, interleukin 6 receptor

Cite as

Pawłowska-Kamieniak A, Krawiec P, Pac-Kożuchowska E.

Interleukin 6: Biological significance and role in inflammatory bowel diseases. Adv Clin Exp Med. 2021;30(4):465-469.

doi:10.17219/acem/130356

DOI

10.17219/acem/130356

Copyright

Copyright by Author(s)

This is an article distributed under the terms of the

Creative Commons Attribution 3.0 Unported (CC BY 3.0)

(https://creativecommons.org/licenses/by/3.0/) 


\section{Introduction}

Cytokines are glycoproteins with molecular weights ranging from a few to a dozen or so kilodaltons (kDa). These molecules affect a number of processes in the living body, such as proliferation, differentiation and migration of cells. Cytokines are produced mainly by immune cells and may act in 3 different manners. They may have an influence on the same cells that produced them (autocrine action), on nearby cells (paracrine action) or on distant cells (endocrine action). ${ }^{1}$ Thus, by affecting many cells, cytokines are mediators of immune reactions and they also participate in hematopoietic processes. Currently, more than 100 distinct types of cytokines are known, and due to continuing progress in the field of immunology, this number is steadily growing. The immune response of a particular cytokine depends on its concentration, presence of appropriate effector cells, and the expression of specific receptors for cytokines on target cells. ${ }^{1-4}$

The currently known types of cytokines include interleukins, interferons, chemokines, superfamily of tumor necrosis factor alfa (TNF- $\alpha$ ) molecules, macrophage migration inhibiting factor (MIF), and transforming growth factor beta $(\text { TGF- } \beta)^{2,4}$

To date, 39 interleukins are known and some of them consist of several subtypes. Based on specific properties, interleukins may be divided into pro-inflammatory and anti-inflammatory cytokines. ${ }^{1,2,4}$

\section{Inflammatory bowel diseases: immunological background}

Inflammatory bowel diseases (IBD) are autoimmune diseases whose incidence and prevalence are increasing worldwide. They are a group of chronic gastrointestinal disorders characterized by multifactorial, still unknown pathogenesis, varied symptomatology, course with periods of exacerbation and remission, and polymorphic infiltration in histopathological examination. They include Crohn's disease (CD), ulcerative colitis (UC) and IBD of unclassified type (IBD-U). ${ }^{2,4-12}$

The etiopathogenesis of these diseases is unknown and multifactorial. There have been several factors suggested to be involved in the development of IBD, including genetic susceptibility, environmental factors and immune response dysregulation. , $, 5,6,8-10,12-14$ The action of environmental factors may lead to damage of the mucosal barrier, development of local inflammatory reaction with the release of many mediators of the inflammatory process, and infiltration of many inflammatory cells. ${ }^{15}$ It is considered that IBD could result from dysregulation of the intestinal barrier and a pathologic activation of the intestinal immune response toward environmental antigens.

It has been shown that $\mathrm{CD}$ results from abnormal response of the intraepithelial $\mathrm{T}$ lymphocytes (Th1), triggered by environmental factors, which induces a cascade of pro-inflammatory cytokines, particularly TNF- $\alpha$. Increased activation of CD4 T cells (Th2) lymphocytes, which are responsible for interleukin production and increased production of antibodies, is observed in UC. ${ }^{16-18}$

It is believed that disorders of intestinal immune mechanisms may be caused by imbalance between pro-inflammatory cytokines and anti-inflammatory cytokines, resulting in a chronic inflammatory process in the intestinal wall. $2,5,6,19$

As it is known, pro-inflammatory cytokines in IBD initiate, intensify and support the development of the inflammatory process in the intestine. In patients with IBD, the intestinal wall is infiltrated with inflammatory cells, including monocytes and macrophages which are the source of pro-inflammatory cytokines. Cytokines, which play a role in the pathogenesis of IBD, may represent a new biomarker for the intensity of inflammatory changes in gastrointestinal tract. $6,7,9,17,18$

\section{Interleukin 6 and its role in transmission of the activation signal}

Interleukin 6 (IL-6) is a prominent cytokine with a pleiotropic effect on inflammation and immunity. Interestingly, IL-6 can both boost and reduce inflammation. It is one of the proinflammatory cytokines and it also has many regenerative and anti-inflammatory properties. ${ }^{20-24}$ The pro- or antiinflammatory effect depends on the target cells and this dual activity of IL- 6 has been the subject of many studies. ${ }^{25}$

Interleukin 6 is a four-helical protein of 184 amino acids. ${ }^{1}$ It is involved in anti-infective response, acute phase reaction and hematopoiesis. It is believed to be one of the main factors regulating the immune defense mechanism of the body. ${ }^{25}$ Interleukin 6 is mainly produced by monocytes and macrophages, but also by endothelial cells, activated Th2 lymphocytes and fibroblasts. ${ }^{15,23,24}$ The main factor inducing the production of IL- 6 is interleukin $1 \beta$ (IL-1 $\beta)$, while less important stimulating factors include TNF- $\alpha$, interferons and lipopolysaccharides (LPS). ${ }^{26}$ Interleukin 6 is also produced during viral infections.

The cytokines are characterized by binding with the appropriate receptors, thereby activating the transmission of the appropriate signal in the effector cell. The interleukin 6 receptor (IL-6R) consists of 2 subunits: a 80-kDa glycoprotein (IL-6R, gp80) and the signal transducing subunit 130-kDa glycoprotein (gp130).,4,23 Interleukin 6 belongs to a family of 10 cytokines, which act through receptor complexes containing the cytokine receptor subunit gp130.15,22,25 In addition to IL-6, members of this family of cytokines include interleukin 11 (IL-11), leukemia inhibitory factor (LIF), ciliary inhibitory factor (CNTF), oncostatin M (OSM), cardiotrophin-1 
(CT-1), cardiotrophin-like cytokine (CLC), neutropoietin (NPN), interleukin 27 (IL-27), and interleukin 31 (IL-31). ${ }^{1,13,20,21,23,24,27,28}$ Gp130 is expressed by most cells in the body, while the IL-6R is mainly expressed by hepatocytes and some leukocytes, such as neutrophils, monocytes and some lymphocytes. As previously mentioned, the pro- or anti-inflammatory effect of IL- 6 depends on the target cells. The dual nature of IL- 6 is associated with different receptor activation: classical and trans-signaling. ${ }^{17,18,20-22,25,27,28}$ Anti-inflammatory messages are transmitted by binding of IL- 6 to IL-6R in the cell membrane and this complex interacts with 2 molecules of gp130, leading to the signal activation. The IL-6-dependent activation through the membranebound IL-6R is called classic signaling. ${ }^{4,13,29}$ Interleukin 6 classical signaling induces the acute-phase response and is considered to have anti-inflammatory effects (Fig. 1). In body fluids, these receptors are present in the soluble form of soluble IL-6 receptor (sIL-6R) and soluble glycoprotein 130 (sgp130). Pro-inflammatory messages are transmitted by binding of IL- 6 to sIL-6R and this process is called trans-signaling (Fig. 2). ${ }^{4,13,29}$ This second activation through binding of complex IL- 6 with sIL-6R induces trans-signaling, also via gp130. This trans-signaling transmission is the primary activation mechanism in chronic inflammatory processes. ${ }^{13,17,25,29,30}$

The subunit of gp130 present in the cell membrane enhances the action of IL-6. The sIL-6R is agonistic and has signal transmission ability in the cell through

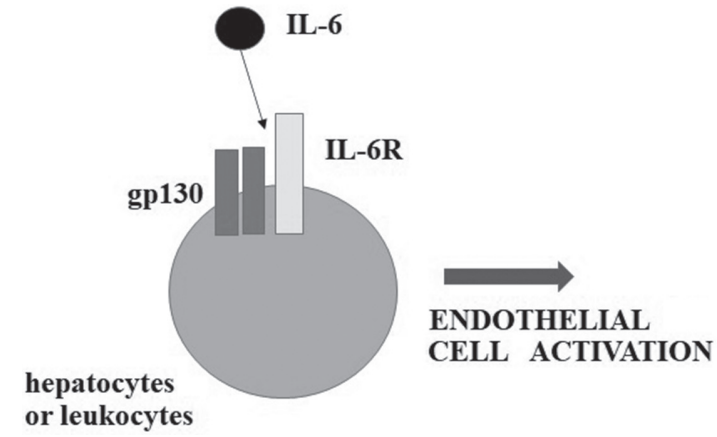

Fig. 1. Classical IL-6 activation (classic signaling)

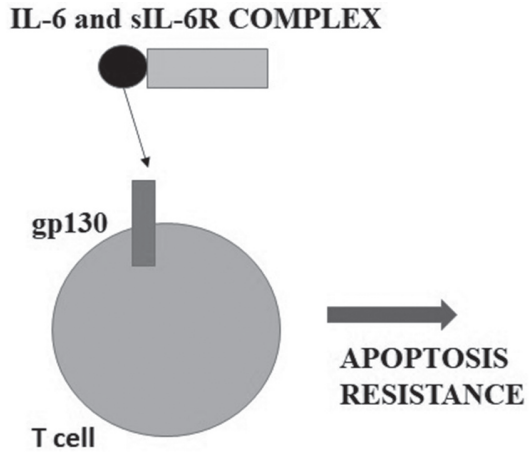

Fig. 2. sIL-6 R-mediated cell signaling (IL-6 trans-signaling)

interaction with the gp130. The IL-6/sIL-6R complex stimulates target cells, which are unresponsive to IL-6 alone due to lack of expression membrane-bound IL-6R. As mentioned earlier, gp130 is expressed in many cells, thus trans-signaling via complex IL-6 and sIL-6R can activate many cells of the body. In both classical signaling and trans-signaling, responses are elicited through the engagement with the membrane-bound gp130, a subunit of IL-6R. . $^{8,25,27,31,32}$

The sIL-6R is the result of the enzymatic action of a disintegrin and metalloproteinase ADAM 10 and ADAM 17 on the IL-6R membrane receptor. In contrast to other cytokines, sIL-6R does not neutralize IL-6, and IL-6/ sIL-6R complexes activate cells, like IL-6 alone. Therefore, the combination of IL- 6 with sIL-6R acts agonistically and intensifies the inflammatory process. Constant stimulation and maintenance of this process leads to tissue damage. ${ }^{1,8,10,25,31,32}$ Under physiological conditions, the transactivation by IL-6/sIL-6R complexes is limited by sgp130, which captures these complexes and prevents their attachment to the membrane gp130. In healthy people, the concentration of sgp130 is significantly higher than the concentration of IL-6/sIL-6R. It is different in autoimmune diseases, which include IBD. Classical IL-6 signaling is unaffected by sgp130, but soluble form of gp130 is the inhibitor of IL- 6 trans-signaling responses (Fig. 3). 1,25,29,33

Apoptosis plays an important role in regulating growth, tissue homeostasis, development and immune responses. The induction of apoptosis is mediated by extrinsic and

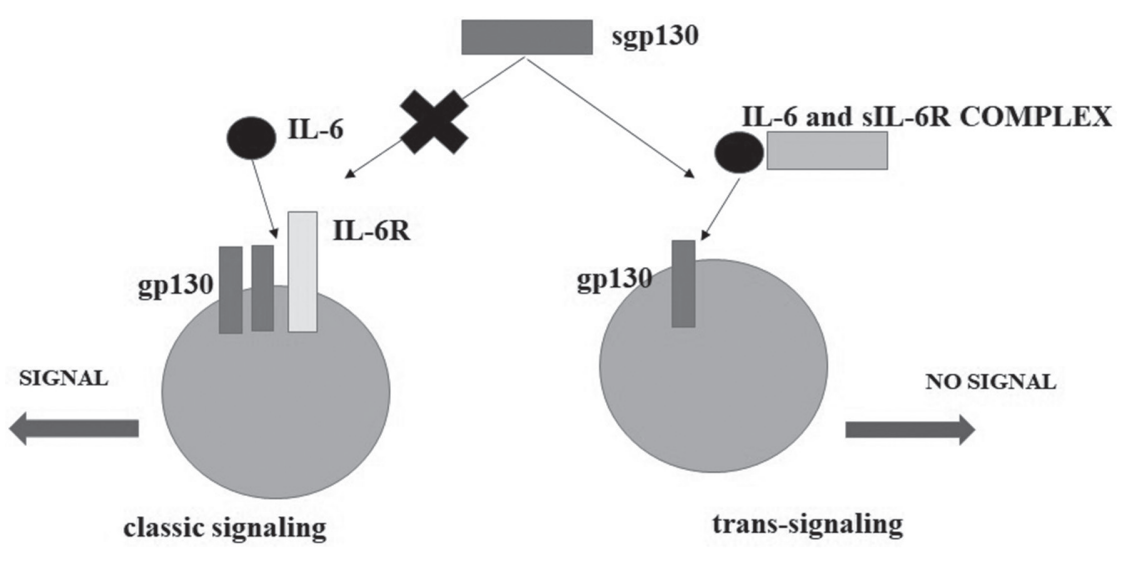

Fig. 3. Selective inhibition of trans-signaling by sgp130 
intrinsic pathways, which require the cooperation of a series of molecules. Trans-signaling response plays important role in resolution of inflammatory responses. ${ }^{30,34}$ Interleukin 6 trans-signaling is involved in the maintenance of the state of chronic inflammatory disease, among others IBD. ${ }^{8,17}$

Interleukin 6 associated with the receptor in trans-signaling process makes use of JAK tyrosine kinases (Janus kinases) and STAT proteins (signal transducers and activators of transcription), and activates the STAT3 transcription factor, which stimulates the inflow of granulocytes and lymphocytes to the intestinal epithelium, inhibits the apoptosis of damaged cells and repairs the intestinal tissue. ${ }^{1,10,11,13,14,27,32,34,35}$ It is believed that the inhibition of T lymphocyte apoptosis is due to STAT3 induction of anti-apoptotic Bcl members, Bcl-2 and Bcl-xL, which leads to the accumulation of these lymphocytes in the tissue and the formation of chronic inflammatory process. ${ }^{1,10,16,30}$ Thus, antibodies against IL-6R, such as tocilizumab, may be used in inhibition of the inflammatory response in rheumatology. $1,9,16,18,28,29,31$

It is well known that one of the basic functions of IL-6 is the influence on the proliferation and differentiation of B lymphocytes into cells releasing immunoglobulins of different classes. Furthermore, it is known that IL-6, together with interleukin 1 (IL-1), is involved in the stimulation of $\mathrm{T}$ lymphocytes recognizing the antigen. ${ }^{4}$ Similarly to IL-1 and interferons, IL-6 plays an important role in inducing a response to a fever. It may also be responsible for the development of many inflammatory conditions and diseases. It participates in the stimulation of the production of acute-phase proteins, involved in the development of an inflammatory response to infection, injury or tissue damage, in the liver. It is believed that in inflammatory states, the concentration of IL- 6 (as well as of acute-phase proteins) in body fluids increases significantly. ${ }^{25,26}$ Interleukin 6 also affects appetite suppression, as the cytokines of the IL- 6 group include leptin, which is an anorexigenic hormone.

Interleukin 6, as mentioned above, is the main factor inducing the synthesis of acute-phase proteins in the liver. Moreover, STAT3 induces liver transcription of the hepcidin, which regulates iron metabolism. Hepcidin inhibits the release of iron from macrophages and other cells, as well as inhibits its absorption in the intestine, which leads to anemia associated with chronic inflammation. ${ }^{10,25,26}$

\section{The participation of interleukin 6 in IBD}

In IBD, the innate immune response plays an important role. It is known that IL-6 signaling plays an important role in maintenance of chronic intestinal inflammation in IBD. Many studies have shown, that IL- 6 is a main inducer of Creactive protein (CRP), and IL-6 and sIL-6R levels are positively associated with elevated levels in IBD. ${ }^{23,33}$ As already mentioned, IL- 6 has been found to use an alternative pathway to activate target cells lacking the membranebound IL-6 receptor through a naturally occurring soluble form of the IL-6R. Although mostly regarded as a proinflammatory cytokine, IL- 6 also has many regenerative functions as an anti-inflammatory cytokine. The availability of IL-6, membrane-bound IL-6R, soluble IL-6R, and gp130 determines the trans- or classic signaling response. Different expression of IL- 6 and sIL-6R in healthy and diseased individuals has been demonstrated. In inflammatory conditions, serum concentration of sIL-6R increases. Increased availability of sIL-6R and a high inflammation-induced concentration of IL- 6 increase the strength of the cellular response towards IL- 6 . In the body, the ratio of sgp130 in contrast to sIL-6R is an antagonist of IL-6-induced signaling. Sgp130 together with sIL-6R act as a buffer system that, in the excess of sIL-6R, favors trans-signaling and, in the excess of sgp130, blocks IL-6-induced classic signaling. ${ }^{21,33}$ Interleukin 6 controls the balance between pro-inflammatory $\mathrm{T}$ cells and immunosuppressive regulatory T cells. The cytokines such as IL- 6 or TNF- $\alpha$ are elevated in most of the inflammatory conditions and therefore have been recognized as targets of therapeutic intervention.

Clinical trials using anti-IL-6 therapy (PF-04236921) by subcutaneous injection in IBD, especially in patients with moderate to severe CD who previously failed treatment with anti-TNF therapy, appear to be promising. ${ }^{35}$ Elevation of IL- 6 concentration can be considered as an early and sensitive, although non-specific, marker for various inflammatory conditions. ${ }^{26,33}$ Diagnosis, monitoring and evaluation of the severity and intensity of intestinal lesions in the course of IBD is based on clinical, endoscopic and histopathological assessment. The aim of many studies was to identify novel biomarkers assessing the intensity of inflammation in the course of IBD.

In many available studies, it has been shown that serum levels of IL-6 are elevated in patients with exacerbation of UC or CD, and decreased in IBD remission. . $^{5,9,11,12,14,19,33}$ Moreover, it has been presented that IL- 6 level correlates with the severity of inflammatory changes in the intestine. ${ }^{2,7}$ A similar relationship, according to CiećkoMichalska et al., exists in patients with CD. ${ }^{5}$ Vasilyeva et al. also showed an increase of IL- 6 levels in adolescent patients with active CD compared to those in disease remission and to the control group. ${ }^{12}$ Lochhead et al. in prospective cohort studies found higher levels of IL- 6 and high-sensitivity CRP (hsCRP) in patients with IBDs. ${ }^{19}$

Nikolaus et al. demonstrated an increase of IL-6 concertation in the active form of both UC and CD, without significant increase of the sIL-6R and sgp130 receptor concentrations. ${ }^{9}$ In a study by Mitsuyama et al., elevated concentrations of both IL- 6 and sIL-6R were found in patients with active forms of UC and CD. ${ }^{8}$ In another study, Mitsuyama et al. demonstrated elevated concentration of IL-6 as well as SIL-6R and sgp130 in the exacerbation of IBD. ${ }^{33}$ Takač et al. demonstrated statistically higher serum levels 
of IL-6 in patients with CD and UC than in a control group. At the same time, no statistically significant differences of IL- 6 were found in both groups of patients. ${ }^{11}$

It is known that the determination of the level of proinflammatory cytokines, including IL-6, may be used in the diagnosis and monitoring of patients with IBD. Several studies have provided evidence for the important role of IL-6 in inflammatory disorders, including IBD. Interleukin 6 has become a non-invasive marker of the assessment of activity and severity of inflammation in the course of IBD. The assessment of serum concentration of IL- 6 and other cytokines, and evaluation of their correlation with IBD activity may contribute to the identification of reliable non-invasive markers of IBD and identification of novel therapeutic targets.

\section{ORCID iDs}

Agnieszka Pawłowska-Kamieniak

(D) https://orcid.org/0000-0002-7747-420X

Paulina Krawiec (D) https://orcid.org/0000-0002-2197-2197

Elżbieta Pac-Kożuchowska (D) https://orcid.org/0000-0002-3204-2235

\section{References}

1. Rose-John S. Interleukin- 6 family cytokines. Cold Spring Harb Perspect Biol. 2018;10(2):a028415. doi:10.1101/cshperspect.a028415

2. Polińska B, Matowicka-Karna J, Kemona H. The cytokines in inflammatory bowel disease [in Polish]. Postepy Hig Med Dosw (Online). 2009; 63:389-394. PMID:19724079

3. Lee SH, Kwon J, Cho ML. Immunological pathogenesis of inflammatory bowel disease. Intest Res. 2018;16(1):26-42. doi:10.5217/ir.2018. 16.1.26

4. Turner MD, Nedial B, Hurst T, Pennington DJ. Cytokines and chemokines: At the crossroads of cell signaling and inflammatory disease. Biochim Biophys Acta. 2014;1843(11):2563-2582. doi:10.1016/j.bbamcr. 2014.05.014

5. Ciećko-Michalska I, Wierzbicka-Tutka I, Szczepanek M, Fedak D, Mach T. Could the cytokines concentration be a marker of IBD activity and be useful in evaluation of IBD differentiation? [in Polish]. Przeg/Lek. 2016;73(5):301-304. PMID:29629745

6. Eder P, Stawczyk K, Krela-Kaźmierczak I, Linke K. Chosen markers of inflammation in inflammatory bowel disease. Gastroentereol Pol. 2007;14(6):429-431.

7. Neubauer K, Bednarz-Misa I, Walecka-Zacharska E, et al. Oversecretion and overexpression of nicotinamide phosphoribosyltransferase/ Pre-B colony-enhancing factor/visfatin in inflammatory bowel disease reflects the disease activity, severity of inflammatory response and hypoxia. Int J Mol Sci. 2019;4;20(1):166. doi:10.3390/ijms20010166

8. Mitsuyama K, Toyonaga A, Sasaki E, et al. Soluble interleukin- 6 receptors in inflammatory bowel disease: Relations to circulating interleukin-6. Gut. 1995;36(1):45-49. doi:10.1136/gut.36.1.45

9. Nikolaus $\mathrm{S}$, Waetzing GH, Butzin $\mathrm{S}$, et al. Evaluation of interleukin- 6 and its soluble receptor components sIL-6R and sgp 130 as markers of inflammation in inflammatory bowel diseases. Int $J$ Colorectal Dis. 2018;33(7):927-936. doi:10.1007/s00384-018-3069-8

10. Sanchez-Muňoz F, Dominguez-Lopez A, Yamamoto-Furusho JK. Role of cytokines in inflammatory bowel disease. World J Gastroenterol. 2008;14(27):4280-4288. doi:10.3748/wjg.14.4280

11. Takač B, MihaljevičS, Štefanić M, Glavas-Obrovac L, Kibel A, Samardzija M. Importance of interleukin 6 in pathogenesis of inflammatory bowel disease. Coll Antropol. 2014;38(2):659-664. PMID:25145003

12. Vasilyeva E, Abdulkhakov $\mathrm{S}$, Cherepnev G, et al. Serum cytokine profiles in children with Crohn's disease. Mediators Inflamm. 2016;2016: 7420137. doi:10.1155/2016/7420127
13. Nguyen PM, Putoczki TL, Ernst M. STAT3-activating cytokines: A therapeutic opportunity for inflammatory bowel disease? J Interferon Cytokine Res. 2015;35(5):340-350. doi:10.1089/jir.2014.0225

14. Carey R, Jurickova I, Ballard E, et al. Activation of an IL-6: STAT3-dependent transcriptome in pediatric-onset inflammatory bowel disease. Inflamm Bowel Dis. 2008;14(4):446-457. doi:10.1002/ibd.20342

15. Xue X, Falcon DM. The role of immune cells and cytokines in intestinal wound healing. Int J Mol Sci. 2019;20(23):6097. doi:10.3390/ ijms20236097

16. Ahluwalia B, Moraes L, Magnusson MK, Öhman L. Immunopathogenesis of inflammatory bowel disease and mechanisms of biological therapies. Scand J Gastroenterol. 2018;53(4):379-389. doi:10.1080/003 65521.2018.1447597

17. Neurath MF.Cytokines in inflammatory bowel disease. Nat Rev/mmunol. 2014;14(5):329-342. doi:10.1038/nri3661

18. Sanchez-Muñoz F, Dominguez-Lopez A, Yamamoto-Furusho JK. Role of cytokines in inflammatory bowel disease. World J Gastroenterol. 2008;14(27):4280-4288. doi:10.3748/wjg.14.4280

19. Lochhead P, Khalili H, Ananthakrishnan AN, Richer JM, Chan AT. Association between circulating levels of C-reactive protein and interleukin- 6 and risk of inflammatory bowel disease. Clin Gastroenterol Hepatol. 2016;14(6):818-824. doi:10.1016/j.cgh.2016.01.016

20. Scheller J, Chalaris A, Schmidt-Arras D, Rose-John S. The pro- and anti-inflammatory properties of the cytokine interleukin-6. Biochim Biophys Acta. 2011;1813(5):878-888. doi:10.1016/j.bbamcr.2011.01.034

21. Rose-John S. IL-6 trans-signaling via the soluble IL-6 receptor: Importance for the pro-inflammatory activities of IL-6. Int J Biol Sci. 2012; 8(9):1237-1247. doi:10.7150/ijbs.4989

22. Baran $P$, Hansen $S$, Waetzig GH, et al. The balance of interleukin (IL)- 6 , IL-6:soluble IL-6 receptor (IL-6R) and IL-6:sIL-6R:sgp130 complexes allows simultaneous classic and trans-signaling. J Biol Chem. 2018; 293(18):6762-6775. doi:10.1074/jbc.RA117.001163

23. Kishimoto T. Interleukin-6: Discovery of a pleiotropic cytokine. Arthritis Res Ther. 2006;8(Suppl 2):S2. doi:10.1186/ar1916

24. Hirano T. Interleukin 6 in autoimmune and inflammatory diseases: A personal memoir. Proc Jpn Acad Ser B Phys Biol Sci. 2010;86(7):717-730. doi:10.2183/pjab.86.717

25. Liu X, Jones GW, Choy EH, Jones SA. The biology behind interleukin- 6 targeted interventions. Curr Opin Rheumatol. 2016;28(2):152-160. doi:10.1097/BOR.0000000000000255

26. Narazaki M, Kishimoto T. The two-faced cytokine IL- 6 in host defense and diseases. Int J Mol Sci. 2018;19(11):3528. doi:10.3390/ijms19113528

27. Scheller J, Ohnesorge N, Rose-John S. Interleukin-6 trans-signalling in chronic inflammation and cancer. Scand J Immunol. 2006;63(5): 321-329. doi:10.1111/j.1365-3083.2006.01750.x

28. Jones GW, Hill DG, Cardus A, Jones SA. IL-27: A double agent in the IL-6 family. Clin Exp Immunol. 2018;193(1):37-46. doi:10.1111/cei.13116

29. Lokau J, Agthe M, Garbers C. Generation of soluble interleukin-11 and interleukin -6 receptors: A crucial function for proteases during inflammation. Mediators Inflamm. 2016;2016:1785021. doi:10.1155/ 2016/1785021

30. Lee MJ, Lee JK, Choi JW, et al. Interleukin-6 induces S100A9 expression in colonic epithelial cells through STAT 3 activation in experimental ulcerative colitis. PLoS One. 2012;7(9):e38801. doi:10.1371/journal. pone.0038801

31. Desreumaux P. Specific targeting of IL-6 signalling pathway: A new way to treat IBD? Gut. 2000;47(4):465-466. doi:10.1136/gut.47.4.465

32. Neuman MG. Signaling for inflammation and repair in inflammatory bowel disease. Rom J Gastroenterol. 2004;13(4):309-316. PMID: 15624029

33. Mitsuyama K, Mitsuyama K, Tomlyasu N, et al. A form of circulating interleukin- 6 receptor component soluble gp130 as a potential interleukin-6 inhibitor in inflammatory bowel disease. Clin Exp Immunol. 2006;143(1):125-131. doi:10.1111/j.1365-2249.2005.02960

34. Lee SD, Choe JW, Lee BJ, et al. Butein effects in colitis and interleukin-6/signal transducer and activator of transcription 3 expression. World J Gastroenterol. 2015;21(2):465-474. doi:10.3748/wjg.v21.i2.465

35. Danese $S$, Vermeire $S$, Hellstern $P$, et al. Randomised trial and openlabel extension study of an anti-interleukin- 6 antibody in Crohn's disease (ANDANTE I and II). Gut. 2019;68(1):40-48. doi:10.1136/gutjnl2017-314562 